\title{
The KM3NeT/ARCA detection capability to a diffuse flux of cosmic neutrinos
}

\author{
Rosa Coniglione* \\ INFN - Laboratori Nazionali del Sud (LNS), Via Santa Sofia 62, 95123 Catania, Italy \\ E-mail: rosa.coniglionedlns.infn.it
}

\section{Luigi Antonio Fusco}

INFN - Sezione di Bologna and Dipartimento di Fisica e Astronomia dell'Universitá di Bologna, Viale Berti-Pichat 6/2, 40127 Bologna, Italy

E-mail: luigiantonio.fusco@bo.infn.it

\section{Antonio Marinelli}

INFN - Sezione di Pisa and Dipartimento di Fisica dell'Universitá di Pisa, Largo B. Pontecorvo 3, 56127 Pisa, Italy

E-mail: antonio.marinelli@pi.infn.it

\section{on behalf of the KM3NeT Collaboration}

The KM3NeT Collaboration aims at the construction of the next-generation large-volume neutrino telescope in the Mediterraean Sea. The planned KM3NeT/ARCA detector will allow the detailed study of cosmic neutrinos both in point-source and in diffuse-flux mode. The foreseen performance in searches for cosmic neutrinos have been reported in the KM3NeT Letter of Intent. Thanks to the use of more sophisticated analysis methods and to the development of a new track reconstruction algorithm, new estimates of the detection capabilities of the ARCA detector for a diffuse flux of neutrinos originating from the whole sky and from a selected region covering the central part Galactic Plane are reported. The detection capability of the detector has been estimated analyzing both the cascade and track channels.

35th International Cosmic Ray Conference - ICRC2017

10-20 July, 2017

Bexco, Busan, Korea

\footnotetext{
* Speaker.
} 


\section{Introduction}

The search of astrophysical high-energy neutrinos adds important pieces to the study of production and propagation of primary cosmic rays (CRs). Neutrinos can be produced at the sources of ultra high-energy cosmic rays (UHECRs) or in the encountered dense environment and then travel to Earth without any deflection or absorption. CRs can produce neutrinos as they interact with matter or radiation via inelastic collisions. As a consequence of these interactions, charged mesons are produced which eventually decay to neutrinos. A diffuse flux of cosmic neutrinos would then originate either from the ensemble of unresolved individual sources, too faint to be detected, or from the interaction of high-energy CRs during their propagation in the Universe. The measurement of the spectrum and flavour composition of a diffuse flux of cosmic neutrinos could provide information on their production mechanisms. This can be also related to the CR acceleration and interaction properties.

In an under-water/ice neutrino telescope two kind of events can be identified: track-like events induced by muon neutrino charged-current interactions and shower-like events induced by neutraland charged-current electron and tau neutrino interactions. Due to the long path travelled by muons in rock and seawater/ice, the selection of track-like events allows to considerably increase the effective detector volume and the events are reconstructed with an excellent angular resolution. Shower-like events are properly reconstructed if the neutrino interaction happens in the matter inside or close to the detector volume. Since they are characterized by a very dense hit pattern close to the neutrino interaction point, a better energy resolution than in the track channel can be achieved.

The analysis of data collected with the IceCube detector have provided the observation of an excess of neutrinos over the expected background $[1,2,3]$. The measured flux can be modeled with single-power-laws $d N_{v} / d E_{v}=\Phi_{0} E_{v}^{-\Gamma}$ with a normalization factor $\Phi_{0}$ and spectral index $\Gamma$. Assuming an astrophysical neutrino flux which is isotropic over the whole sky and equally distributed in the three neutrino flavours at Earth, the combined analysis of the IceCube data sample yields a best-fit spectral index $\Gamma=2.50 \pm 0.09$ and a normalization factor at $100 \mathrm{TeV}$ $\Phi_{0}^{3 f}(100 \mathrm{TeV})=6.7_{-1.2}^{+1.1} \cdot 10^{-18} \mathrm{GeV}^{-1} \mathrm{~cm}^{-2} \mathrm{~s}^{-1} \mathrm{sr}^{-1}$ for an all-flavour flux (3f) [2]. A separate measurement of muons induced by neutrinos coming from the Northern hemisphere in IceCube [3] reported a best-fit, single-flavour flux $\Phi_{0}^{1 f}(100 \mathrm{TeV})=9.0_{-2.7}^{+3.0} \cdot 10^{-19} \mathrm{GeV}^{-1} \mathrm{~cm}^{-2} \mathrm{~s}^{-1} \mathrm{sr}^{-1}$ and $\Gamma=2.13 \pm 0.13$ [3]. These measurements of the cosmic neutrino flux by IceCube might be a hint to the presence of multiple components in the IceCube signal, such as a hard/extragalactic plus a soft/galactic component [4].

The KM3NeT/ARCA detector, being in the Mediterranean Sea, will allow for a study this signal with high precision, thanks to it large effective area, excellent background rejection and unprecedented reconstruction performance. The detector will be made of 2 building blocks to be deployed at a depth of about $3500 \mathrm{~m}$ off-shore Capo Passero, Sicily. Each of them will host 115 detection units (DU), vertical strings kept taut by top buoys, each holding 18 digital optical modules (DOMs). The DOM is a pressure resistant glass sphere hosting 31 3-inch photomultiplier tubes (PMTs) and the off-shore electronics. In the ARCA configuration, DUs will be spaced by $90 \mathrm{~m}$ and DOMs on each string will be $36 \mathrm{~m}$ apart. The strings are connected to a junction box which allows the communication to shore and the distribution of power to the apparatus via a 100 
km-long electro-optical cable to the shore station located in Portopalo di Capo Passero, Sicily. The first ARCA detection units are now installed [5]. A detailed description of the apparatus can be found in [6]

The detection and the detailed investigation of the astrophysical flux observed by IceCube is one of the main physics goals of the KM3NeT/ARCA telescope [6]. Updates of the searches for diffuse fluxes of cosmic neutrinos from all-sky and from a selected region of the Galactic Plane will be reported in this contribution. Moreover, for the first time the performance of KM3NeT/ARCA to neutrinos from a selected region of the Galactic Plane is here reported for the cascade channel.

Thanks to a new more efficient trigger algorithm, to new track reconstruction algorithms that provides a better angular and energy resolution, to a pseudo-vertex reconstruction and to a higher efficiency [7] the preliminary results presented here show better values with respect to those presented in [6]. A multivariate analysis (MVA) has been applied to the all-sky search with tracks.

\section{Search method}

To evaluate the sensitivity of KM3NeT/ARCA to neutrino diffuse fluxes detailed Monte Carlo (MC) simulations have been performed. The analysis takes into account (anti-)neutrinos of all flavours $\left(v_{\mu}, v_{e}\right.$ and $\left.v_{\tau}\right)$ in equal proportions and their $\mathrm{CC}$ and $\mathrm{NC}$ interactions. Algorithms reconstructing the direction and energy under the hypothesis of a muon track or a shower have been applied to simulated events [7].

In order to search for a diffuse flux of cosmic neutrinos, the atmospheric background must be evaluated. The largest signal in a neutrino telescope is given by downward-going atmospheric muons, produced in CR air cascades. Their event rates are largely dominant with respect to atmospheric neutrinos produced in the same CR cascades, and to cosmic neutrinos, whose flux is lower by many orders of magnitude. Monte Carlo simulations based on the MUPAGE code [8,9] are used to evaluate the contribution from atmospheric muons. The contribution of atmospheric neutrinos is estimated using the computation of the conventional component by Honda et al. [10] and the prompt component by Enberg et al. [11]. The atmospheric neutrino spectrum is also corrected to account for the CR primary knee as from the H3a estimation of spectrum and composition [12].

The all-sky one flavour cosmic flux is parameterised as follows:

$$
\Phi_{v}\left(E_{v}\right)=1.2 \times 10^{-8}\left(\frac{E_{V}}{G e V}\right)^{-2} \exp \left(\frac{-E_{v}}{3 P e V}\right) \mathrm{GeV}^{-1} \mathrm{~cm}^{-2} \mathrm{~s}^{-1} \mathrm{sr}^{-1}
$$

or

$$
\Phi_{v}\left(E_{V}\right)=4.11 \times 10^{-6}\left(\frac{E_{V}}{G e V}\right)^{-2.46} \mathrm{GeV}^{-1} \mathrm{~cm}^{-2} \mathrm{~s}^{-1} \mathrm{sr}^{-1}
$$

to test different possibilities to fit the IceCube signal. These fluxes were also considered as benchmark fluxes in [6] and are fully compatible with the more recent IceCube measurements of [3] and [2].

The Galactic plane is a promising potential source region of a diffuse astrophysical neutrino flux and the KM3NeT/ARCA detector, located in the Northern hemisphere, is at the optimal position for the observation of neutrinos from the Galactic Plane near the Galactic Center region. In [13] a phenomenological model for the diffuse galactic neutrino emission in the central Galactic 
Plane or Galactic Ridge, related also to the observed IceCube high-energy neutrino events, have been proposed. In this model a nonuniform cosmic-ray (CR) transport scenario with a radially dependent diffusion coefficient has been adopted to explain the high-energy diffuse $\gamma$-ray emission along the whole Galactic Plane, as well as the hardening of CR spectra measured at $250 \mathrm{GeV}$ and the possible cutoffs of Galactic CRs.

For what concerns the flux from the Galactic Plane, a region encompassed by $|\ell|<30^{\circ}$ and $|b|<4^{\circ}$ in galactic coordinates is considered. The neutrino signal from this region has been computed according to the $\mathrm{KRA}_{\gamma}$ model of [13], assuming a $50 \mathrm{PeV}$ cutoff for $\mathrm{CR}$ primaries. The corresponding one-flavour flux from the Galactic Plane region is parameterized here as:

$$
\Phi_{v}\left(E_{V}\right)=5 \times 10^{-6}\left(\frac{E_{V}}{G e V}\right)^{-2.3} \exp \left(\sqrt{\frac{E_{V}}{P e V}}\right) \mathrm{GeV}^{-1} \mathrm{~cm}^{-2} \mathrm{~s}^{-1} \mathrm{sr}^{-1}
$$

Sensitivity studies for KM3NeT/ARCA in the search for diffuse fluxes of neutrinos are based on a maximum-likelihood method applied to the event sample resulting from an event preselection. This allows to calculate the sensitivity and the discovery potential at different significance levels. Loose cuts are in general applied before the likelihood maximisation in order to retain the maximum possible information; thus the resulting event sample is very broad. The likelihood ratio (LR) function:

$$
L R=\sum_{k=1}^{n} \log \frac{\frac{n_{s i g}}{n} P_{s i g}\left(X_{k}\right)+\left(1-\frac{n_{s i g}}{n}\right) P_{b k g}\left(X_{k}\right)}{P_{b k g}\left(X_{k}\right)}
$$

is employed. In this, $n_{\text {sig }}$ is the estimated number of signal events, $n$ is the total number of events and $n-n_{s i g}=n_{b k g}$ is the number of background events. $\mathrm{P}_{s i g}$ and $\mathrm{P}_{b k g}$ are the probability density (PDF) functions for signal and background events, respectively. These PDFs are based on quantities which allow signal/noise separation, as for example the output of the multivariate analysis discriminator or the reconstructed event energy. The LR is maximised by changing $n_{\text {sig }}$ to obtain $L R_{\max }$. Pseudo-experiments (PEs) are performed and LR is maximised for each PE. The distributions of LR max when simulated signals events are present are compared to distributions in the background-only case to evaluate the significances of each simulated observation. The details of the event selection and of the LR maximisation will be presented in the following sections for each analysis channel.

\section{The track channel}

In the analysis based on the selection of track-like events, the main challenge is the identification of tracks originated by neutrinos from the tracks due to atmospheric muons. In the analysis presented here, the traditional approach of using the Earth as a shield is used: events reconstructed with the tracking algorithm as coming from below the horizon (or a few degrees above it) are selected. After this preselection cut, a further rejection of the atmospheric neutrino background and of wrongly-reconstructed atmospheric muons from muons induced by the interaction of astrophysical neutrinos is needed. A Random Decision Forest (RDF), from the Scikit-learn multivariate analysis package [14], is then used for this purpose. The machine learning algorithm is trained on three MC data samples: atmospheric neutrinos, atmospheric muons and neutrinos from signal. In 
the training phase, to classify the events, the algorithm uses a set of "features" that are associated to each events. In this analysis these features are the output parameters of the track reconstruction algorithm and are related to the quality of the reconstructed track or energy or to the event topology. In particular for each event 11 features are considered. The most important features for the rejection of atmospheric muons are the reconstructed zenith angle, the coordinates of the reconstructed pseudo-vertex and the track likelihood. The reconstructed muon energy plays an important role in the rejection of atmospheric neutrinos. The PDFs used to maximize the likelihood (eq. 2.4) are functions of the outputs of the Scikit-learn algorithm and in particular on the probability assigned to each events to be signal. This procedure has been applied to samples of events with different preselection cuts on the reconstructed zenith angle (up to $30^{\circ}$ above the horizon). Similar values were achieved exploring the angular region from $5^{\circ}$ to $20^{\circ}$ above the horizon. To speed up the maximization of LR (eq. 2.4) and provide an adeguate number of pseudo-experiments the $\theta_{\text {rec }}^{z e n}<85^{\circ}$ was chosen. The preliminary results of this analysis are reported in Fig.1 left.
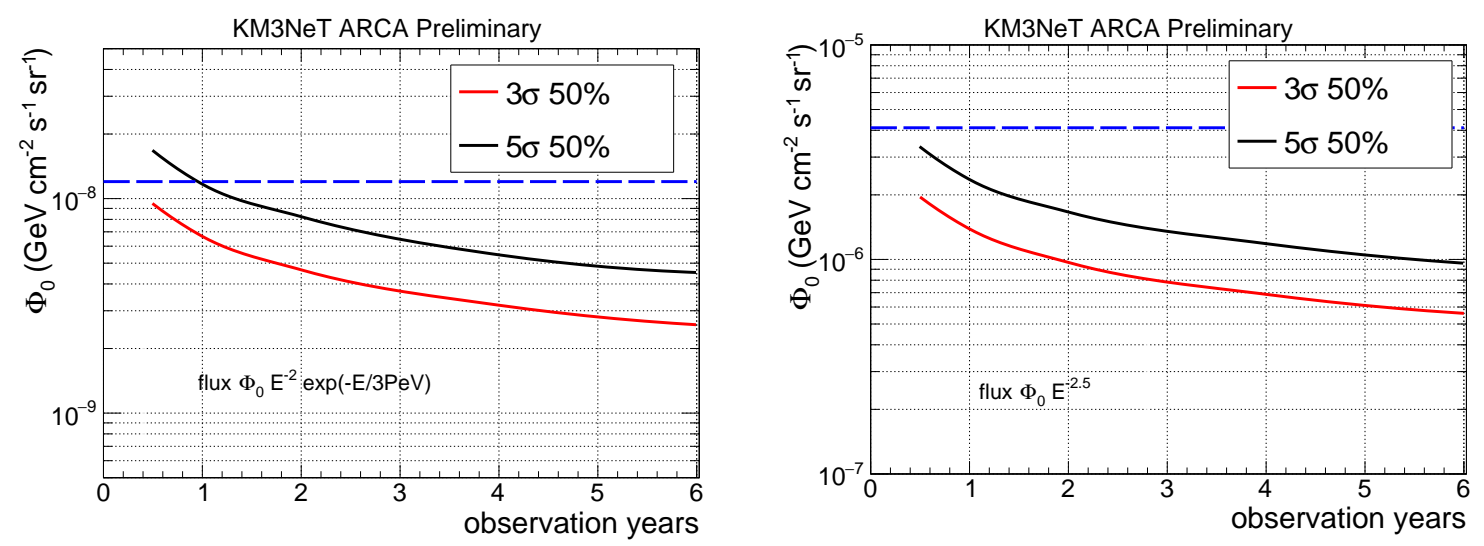

Figure 1: Discovery fluxes as a function of the observation years for the track channels for the ARCA detector for the all-sky analysis for the flux of eq. 2.1 (left) and eq. 2.2 (right). The dashed blue lines represent the normalization factors of eq. 2.1 and 2.2.

With the same procedure the flux of eq. 2.2 was also tested. For this flux the preselection cut has not been optimized and the same selection on the zenith angle $\left(\theta_{\text {rec }}^{z e n .}<85^{\circ}\right)$ was applied as for the previous case. The results are reported in Fig. 1 right. The discovery at $5 \sigma$ can be reached in about one year for a flux with a spectrum $\propto E^{-2}$ and a cutoff at $3 \mathrm{PeV}$ (eq. 2.1) and in less than one year for a softer spectrum $\propto E^{-2.5}$ (eq. 2.2).

To predict the performance of KM3NeT/ARCA to the Galactic Plane region with $|\ell|<30^{\circ}$ and $|b|<4^{\circ}$ an analysis similar to that one reported in [6] has been performed. The results are reported in Fig. 2 The better angular and energy resolution of the track algorithm, that reduces the number of mis-reconstructed muons, together with the better efficiency improves the results. The $5 \sigma$ discovery can be reached in 4 years of operation.

\section{The shower channel}

Also cascade-like events can be exploited to test diffuse flux signals. This was clearly proved in the KM3NeT Letter of Intent: a cosmic flux such as that of eq. 2.1 could be observed with 
high significance in a short amount of time with the full KM3NeT/ARCA detector. In addition, the angular resolution of the experiment for reconstructed cascade-like events is foreseen to be of the order of a couple of degrees when the reconstructed events are above some tens of $\mathrm{TeV}$ [7]. This is actually enough to test also an enhanced neutrino emission coming from a selected region of the Galactic Plane. In addition, the accurate estimation of the neutrino energy - as the resolution is of the order of $10 \%$ - allows for a good discrimination between low-energy/atmospheric neutrinos against the high-energy/cosmic ones.

In order to remove the large background of atmospheric muons, at first only contained events are selected. The containment selection is based on the reconstructed pseudo-vertex from the tracking algorithm described in [6], which is obtained by back-tracking the earliest hits along the reconstructed track direction. In the case of atmospheric muons, these earliest hits are on the outer edge of the detector, thus the pseudo-vertex is usually outside the instrumented volume. For showerlike events, on the other hand, most of the hits are contained within the apparatus. Events with reconstructed pseudo-vertex inside the instrumented volume were selected. Then, according to the reconstructed zenith angle from the tracking algorithm, events are classified as upward-going (sample "A", $\cos \theta_{\text {track }}>-0.4$ ) or downward-going (sample "B", $\cos \theta_{\text {track }}<-0.4$ ). Because of the different nature of the dominant backgrounds in these samples, slightly different selection cuts are applied in the following selection steps. In order to provide a further rejection of low-energy tracks in the sample, a cut on the total ToT ${ }^{1}$ of the event is applied. The hits considered for the computation of this quantity are only those which are in space-time correlation with the reconstructed vertex. This allows to reduce the influence of low-energy track-like events because for this event topology fewer hits are selected with respect to the case of a real cascade-like event. For sample "A" a cut on the total ToT at $3 \mu$ s is applied.

This allows for the reduction of the influence of track-like events because for this event topology fewer hits are selected with respect to the shower assumption than in the case of a real cascadelike event. For sample "B" a more stringent cut on this variable is applied (at $4 \mu \mathrm{s}$ ), while also requiring that the track quality parameter is low. This stricter selection is necessary to reduce the amount of downward going atmospheric muons in the sample.

Once the final event samples are selected, two separate RDFs (one for sample "A" and one for sample "B") are trained to discriminate signal from background events. In this case the signal is given by electron neutrino events, while the background by the residual atmospheric muon contamination. The cut $R D F_{\text {prob }}>0.9$ in the RDF output provides the necessary discrimination between neutrinos and atmospheric muons. Then, the reconstructed energy is used to separate the signal from the background of atmospheric neutrinos as PDF in the likelihood ratio of eq. 2.4. This strategy is optimal for the case of neutrinos from the Galactic Plane, since the restriction of the analysed sky also reduces the amount of background muons in the sample. On the other hand, when looking at the whole sky, a harder selection on the total ToT is necessary - as proved in the KM3NeT Letter of Intent [6].

If one considers events that pass the full event selection, with reconstructed energy larger than $10 \mathrm{TeV}, 6.1$ events per year for the signal from the selected Galactic Plane region are expected, when

\footnotetext{
${ }^{1} \mathrm{KM} 3 \mathrm{NeT}$ PMT hits are recorded via the start time and the duration of the signal above a predefined threshold (time-over-threshold, or ToT). The total ToT associated to each event is the sum over all the causally connected hits selected by the cascade reconstruction algorithm.
} 
about 3.8 events should come from atmospheric background. As a result of the LR maximisation procedure, it is possible to state that the central Galactic Plane flux of eq. 2.3 can be tested with high significance in the shower channel. The sensitivity of KM3NeT/ARCA will be enough to study this flux within less than a year of data taking with the complete detector, and a $5 \sigma$ discovery of such a flux can be obtained in 4 years of operation as shown in Fig.2.

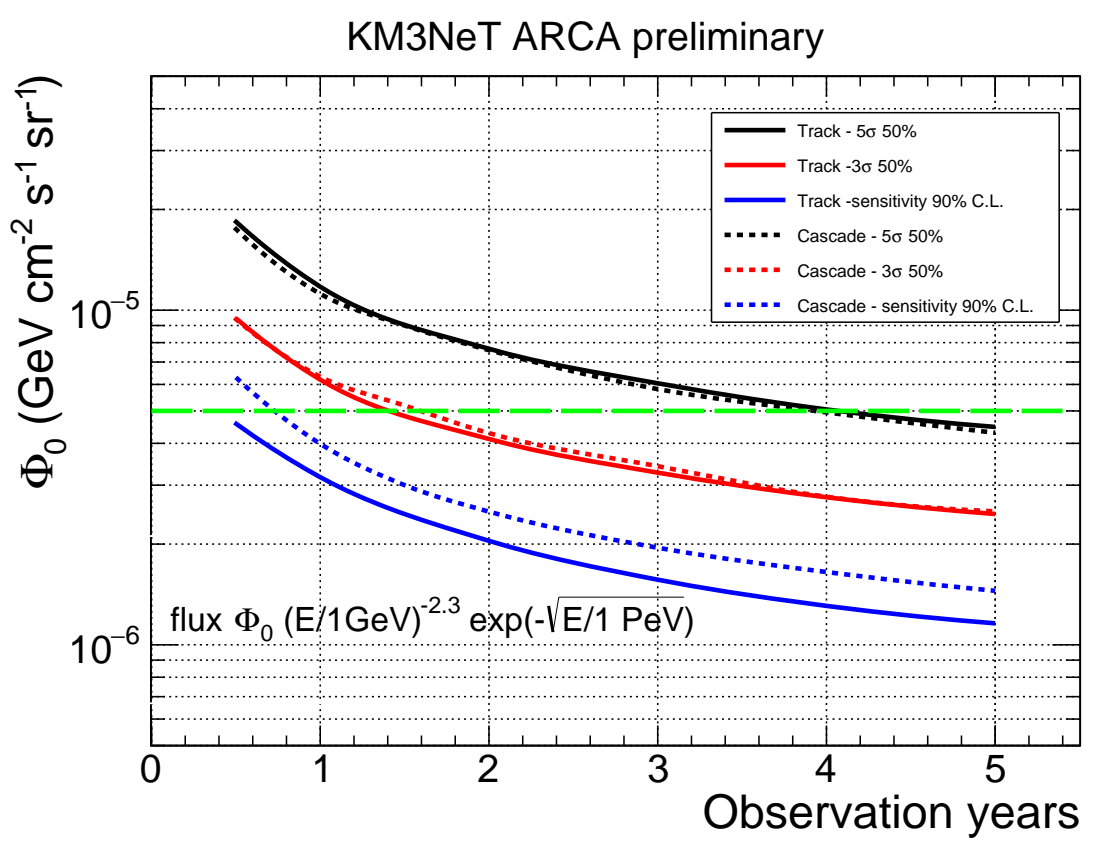

Figure 2: Discovery fluxes and sensitivity as a function of the observation time for the Galactic Plane region $\left(|\ell|<30^{\circ}\right.$ and $|b|<4^{\circ}$ ) for the cascade (dashed lines) and track channels (full lines) for the ARCA detector. The dashed green line corresponds to the normalization factor of eq. 2.3.

\section{Conclusions}

The presence of a multicomponent spectrum in the observed diffuse astrophysical neutrino flux has bcome more and more evident. The extragalactic component is expected to be described by a spectrum with a shape $E^{-\gamma}$ with $\gamma \sim 2$ while the Galactic component with a spectrum $E^{-\gamma}$ with $\gamma \sim 2.5$. Spectra with this shape have been taken into consideration as benchmark fluxes for the evaluation of the detection capability of KM3NeT/ARCA to an all-sky diffuse flux. Expectation of the KM3NeT/ARCA detector for these fluxes were reported for the first time in the KM3NeT Letter of Intent [6]. Some of the results reported in the LoI have been now updated and preliminary results are presented in this work.

A new track reconstruction algorithm has been developed [7]. This algorithm provides a better angular and energy resolution, an improved efficiency and also the reconstruction of the track pseudo-vertex. Moreover, a sophisticated multivariate analysis based on the Scikit-learn packaged allows for the simultaneuos exploitation of the reconstruction output parameters and of parameters related to the topology of the events. The discovery at $5 \sigma$ can be reached after one year of observa- 
tion in the track channel for a flux $\propto E^{-2}$ and a cutoff at $3 \mathrm{PeV}$ (eq. 2.1) and in less than one year for a softer neutrino spectrum $\propto E^{-2.5}$ (eq. 2.2).

Also the expectations for a diffuse high-energy neutrino flux from a Galactic Plane region near the Galactic Center $\left(|\ell|<30^{\circ}\right.$ and $\left.|b|<4^{\circ}\right)$ has been reported for the track channel and for the first time for the cascade channel. In both the channels after four years of observation the neutrino flux predicted in [13] can be discovered with a significance larger then $5 \sigma$. Combining the results of the cascade and track analyses this result can be further improved.

The results shown in this work indicate that KM3NeT can observe the expected high energy diffuse neutrino fluxes with a high significance with few year of observation. Moreover, thanks to its good angular resolution for tracks (less than $0.1^{\circ}$ for $E_{v}>100 \mathrm{TeV}$ ) the observed neutrinos can better discriminate the emitting region in the sky thus helping in the understanding of the origin of astrophysical neutrinos.

\section{References}

[1] M.G. Aartsen et al., Phys. Rev. Lett. 113: 101101 (2014).

[2] M.G. Aartsen, et al., Astrophys. J. 809: 98 (2015).

[3] M.G. Aartsen et al., Astrophys. J. 833 no.1: 3 (2016).

[4] A. Palladino, M. Spurio and F. Vissani, J. Cosmol. Astropart. Phys. 16(12): 045 (2016).

[5] Measurement of the depth dependence of coincidence rates induced by atmospheric muons with the first two KM3NeT Detection Units, M. Jongen for the KM3NeT coll. in these proceedings.

[6] S. Adrián-Martínez et al., Journal of Physics G: Nuclear and Particle Physics, 43 (8), 084001, 2016

[7] KM3NeT/ARCA Event Reconstruction Algorithms - K. Melis for the KM3NeT coll. in these proceedings.

[8] Y. Becherini et al., Astrop. Phys. 25: 1 (2006).

[9] G. Carminati et al., Comput. Phys. Commun. 179: 915-923 (2008).

[10] M. Honda et al., Phys. Rev. D 75: 043006 (2007).

[11] R. Enberg et al., Phys. Rev. D 78: 043005 (2008).

[12] T.K. Gaisser, T. Stanev, and S. Tilav, arXiv:1303.3565 (2013).

[13] D. Gaggero et al., Astrophys. Journ. Letters 815: L25 (2015).

[14] F. Pedregosa et al., Journal of Machine Learning Research 78: 2825-2830 (2011).

[15] S.R. Kelner, F.A. Aharonian and V.V. Bugayov, Phys. Rev. D 74 (3): 034018 (2006).

[16] K. Mannheim, R. Protheroe and J.P. Rachen, Phys. Rev. D 63: 023003 (2001). 